\title{
Transient Non-Suppressible Cough at Altitude Occurring With Cessation of Exercise
}

\author{
Andrew T. Taylor
}

\begin{abstract}
The aim of this report is to describe two subjects with an uncontrollable dry cough that occurred at altitude immediately after cessation of intense exercise; the cough occurred at 5 - 15 second intervals and resolved in 20-30 min with rest or a reduced level of exercise. The presence of cough did not foreshadow an increased risk of acute mountain sickness (AMS) or high altitude pulmonary edema (HAPE) and, in a third subject, was observed to occur at sea level. Its etiology is uncertain but it is probably not related to the cold dry air at altitude, bronchoconstriction, respiratory tract infection, AMS, vasomotor rhinitis or water loss from the respiratory tract. A plausible hypothesis is a transient overload of normal physiologic processes resulting from an imbalance between the increased production of transpulmonary filtrate due to elevated pulmonary artery pressures from intense exercise and hypoxia combined with a sudden reduction in the rate of lymph clearance due to the decrease in hyperpnea following the abrupt cessation of intense exercise. Clinical pulmonary edema results from a breakdown of normal homeostatic processes for removal of interstitial fluid; for individuals in this report, normal homeostatic processes were still operative. Regardless of the mechanism, this syndrome appears to be benign and when it occurs at altitude, it does not imply an increased risk of AMS or HAPE, does not require treatment and does not necessitate a restriction in activity or a prohibition against higher ascent.
\end{abstract}

Keywords: HAPE; High altitude pulmonary edema; Cough; Altitude; Pulmonary lymph flow; Non-suppressible cough

\section{Introduction}

With the growth of ecotourism and global adventure travel,

Manuscript accepted for publication March 19, 2013

Emory University School of Medicine, Department of Radiology and Imaging Sciences, 1364 Clifton Rd, NE, Atlanta, GA 30322, USA. Email: ataylor@emory.edu

doi: http://dx.doi.org/10.4021/jmc1250e ever increasing numbers of people of all ages are now trekking and climbing to very high and even extreme altitudes. As an individual ascends to ever increasing heights, the risk of high-altitude illnesses including the potentially fatal syndrome of high-altitude pulmonary edema (HAPE) also increases. Early diagnosis of HAPE may be difficult since many of the early symptoms (shortness of breath, tachypnea, tachycardia, reduced arterial saturation, fatigue and dry cough) are often present in unaffected climbers at higher altitudes, particularly in cold, dry or dusty environments [13]. Nevertheless, early diagnosis is critical. In the appropriate setting, decreased exercise performance and a dry cough should raise the suspicion of high-altitude pulmonary edema.

In remote locations, subjects with a suspicion of early HAPE should minimize physical activity; continued ascent should not be permitted until symptoms have fully resolved and descent may be indicated. Since many climbing/trekking groups travel on a fixed schedule, these measures may have the effect of separating a client from the group or causing a client to rearrange or terminate his/her trip. The purpose of this report is to describe a syndrome characterized by an exercise induced, non-suppressible dry cough associated with altitude which might be mistaken for early HAPE; this syndrome is hypothesized to represent a short delay in achieving homeostasis and its presence does not require a limitation of activity or a delay in further ascent.

\section{Case Report}

\section{Case 1}

A fit 57-year-old male developed a non-suppressible, dry cough at $5-15$ second intervals after stopping to view Mount Everest part way up the 600 meter hill to Namche Bazaar $(3,440 \mathrm{~m})$. Wheezing was not observed and there was no respiratory stridor. He had started up the hill later than his companions and had been exercising strenuously to catch up. He lived at $250 \mathrm{~m}$, had spent two days in Kathmandu (1,400 $\mathrm{m})$ and the previous night in Phakding $(2,852 \mathrm{~m})$. He was taking acetazolamide $125 \mathrm{mg}$ bid and had no symptoms of fatigue, headache, nausea or sleep disturbance the previous 
night. In spite of an uncontrollable urge to cough every 10 - 15 seconds, he resumed hiking up the hill but slowed his rate of ascent. His symptoms resolved in $20-30$ minutes. Later during the trek, he hiked rapidly with 3 companions from Deboche $(3,820 \mathrm{~m})$ to Pheriche $(4,240 \mathrm{~m})$. On arrival in Pheriche, he jogged up the nearby hillside to the point of exhaustion, took a panorama picture of the valley and then hurried back to join his companions who were waiting to descend to Tengboche. Shortly after joining his companions, the non-suppressible dry cough recurred; in spite of the cough, he started downhill with his companions and the cough resolved in 20 - 30 minutes. There were no other episodes of dry cough during the trip and no symptoms of AMS.

\section{Case 2}

A 58-year-old male had been in Europe on business. Afterwards, he spent three days hiking above Zermatt $(1,620 \mathrm{~m})$ and then traveled to Chamonix $(1,030 \mathrm{~m})$. The next morning he took the cable car to the Aiguille du Midi, spent the afternoon practicing crevasse rescue and then hiked rapidly up to the Cosimiques hut $(3,613 \mathrm{~m})$ jogging the last 100 meters trying to beat his companions to the hut. Within minutes of arrival at the hut, he developed a non-suppressible, hacking dry cough every 10 - 15 seconds. There was no wheezing or history of asthma. The cough resolved in $20-30$ minutes and he had no symptoms of AMS. The following day, he successfully summited Mont Blanc without further symptoms and returned to Chamonix.

\section{Case 3}

A fit 59-year-old male en route to Nepal had a delayed flight arrival in Bangkok. When he deplaned, he had only a few minutes to make his connection for Kathmandu at the opposite end of the terminal. He raced to his gate carrying a 30 pound pack, barely making his flight. After being seated, he developed a non-suppressible dry cough at approximately 10 second intervals which resolved in $20-30 \mathrm{~min}$. There was no wheezing or history of asthma. He later climbed Island Peak $(6,180 \mathrm{~m})$ without symptoms of AMS or recurrence of the cough.

\section{Discussion}

These four episodes of an uncontrollable urge to cough have several elements in common. Three of the four episodes occurred at altitude. They all occurred immediately after the conclusion of intense exercise, not during exercise; they were all associated with a dry, hacking cough at $5-15 \mathrm{sec}-$ ond intervals, all resolved in 20 - 30 minutes, were unassociated with symptoms of AMS and were not predictive of subsequent AMS or pulmonary edema with continuing exercise or ascent to higher elevations. Experienced high altitude medicine physicians will likely have no difficulty distinguishing this syndrome from early HAPE. Most trekking groups, however, are not accompanied by experienced high altitude physicians although physicians and other medical professions may be members of the trekking party and may be called upon for medical advice even though they lack specific training in high altitude. An important factor distinguishing this syndrome from early HAPE is the fact that all of the affected individuals maintained good exercise tolerance in spite of the cough. Although the syndrome appears to be uncommon, when it occurs at altitude, it should be recognized as benign; it does not require treatment, necessitate a restriction in activity or require a prohibition or a delay in ascending to higher altitude.

Common causes for altitude related cough include the cold, dry air which characterizes the high altitude environment as well as a number of other etiologies and conditions such as bronchoconstriction, respiratory tract infection, AMS, vasomotor rhinitis or water loss from the respiratory tract [4]. Cold dry air is an unlikely explanation since cough occurs in long duration hypobaric chamber studies under a controlled temperature and humidity [4], one of the episodes occurred in the Bangkok airport and cough did not occur in the affected individuals when they ascended to higher elevations where the air was likely colder and drier. Respiratory tract infection and vasomotor rhinitis cannot explain the isolated occurrence, acute onset and very short duration of symptoms. Bronchoconstriction is also an unlikely explanation since none of the affected individuals had a history of asthma, wheezing was not observed, symptoms did not appear until the cessation of exercise and symptoms abated without therapy and, in two cases, with continuing but a lower level exercise. Likewise, water loss from the respiratory tract is not a sufficient explanation for these findings since controlled studies in post-exercise, cough prone individuals showed that hyperpnea in cold air increased the cough frequency over baseline by only one cough every two minutes, a frequency insufficient to explain these observations [5].

Up to $65 \%$ of healthy humans will show signs of pulmonary edema triggered by exhaustive maximum effort exercise $[6,7]$. In normal individuals, the increase in pulmonary artery pressures induced by exercise and hypoxia [8], the increase in capillary permeability resulting from hypocapnia [9], and the exercise induced increase in pulmonary capillary blood volume and cardiac output [10] all contribute to a transvascular filtrate into the lung parenchyma which is cleared via lung lymphatic flow [11]. During ascent, the rate of filtration into the interstitial space and the alveoli increases slowly as pulmonary artery pressure increases with exercise and with the increasing degree of hypoxia [11]. The presence of increased pulmonary extravascular fluid has been demonstrated by indirect techniques in $74 \%$ of 262 consecutive climbers of Monte Rosa (4,559 m) [12] and 
episodes of HAPE have occurred in experienced, successful high-altitude climbers [11]. These studies have led investigators to conclude that the risk of HAPE likely exists for most climbers if the rate of ascent and degree of physical effort are great enough $[11,12]$.

Although $74 \%$ of 262 consecutive climbers of Monte Rosa presented with an increase pulmonary extravascular fluid, only one had to be evacuated for HAPE [12] and the possibility exists that, for the large majority of climbers, the increase in pulmonary extravascular fluid noted one hour after arrival at the hut represented a normal physiologic response to the conditions of hypoxia, hypocapnia and exercise observed at a single time point. An increase in pulmonary extravascular fluid is hypothesized to have contributed to the four episodes of post-exercise, non-suppressible cough in this report.

Experimental data suggest a mechanism for the transient, post-exercise non-suppressible cough. During strenuous exercise in normal animals, intravascular fluid rapidly filters into the lung parenchyma in large amounts yet the lung does not develop overt pulmonary edema $[13,14]$. In sheep exercising to exhaustion, pulmonary artery pressures almost double while lung lymph flow increases by a factor of 5 immediately returning to baseline levels when exercise is stopped $[11,14]$. If the sheep are subjected to prolonged exercise, however, lung lymph flow is slower in returning to the baseline value suggesting hydration of the lung interstitium [14]. Hyperpnea is a major mechanism for interstitial fuid clearance in exercising sheep and may be largely responsible for preventing pulmonary edema that might otherwise occur at the high microvascular pressures of strenuous exercise [15]. The basis of the non-suppressible cough may have been the sudden cessation of intense exercise accompanied by a rapid reduction in the rate and depth of ventilation such that lung lymph flow could no longer remove interstitial fluid rapidly enough to prevent fluid from leaking into the alveolar spaces and triggering a non-suppressible cough response that lasted until the fluid could be cleared.

The compressive phase of a cough follows an initial inspiratory phase and may serve to reduce alveolar fluids. After inhaling a volume of air, the glottis is closed and an expiratory effort ensues. Glottic closure maintains lung volume as intrathroacic pressures are building; high intrathoracic pressures developed during glotiic closure may be as great as $300 \mathrm{mmHg}$ [16]. In the subjects of this report, the increased pulmonary alveolar pressures induced by cough relieved symptoms for a few seconds before the uncontrollable urge to cough recurred. Studies in dogs with high pressure pulmonary edema showed that positive end-expiratory pressure improves gas exchange and lung mechanics and that this improvement was associated with redistribution of lung water to the "extra-alveolar" interstitial space [17]. The effect of cough may have also been to help redistribute lung water to the extra-alveolar interstitial space where it could eventually be removed by lung lymphatic drainage. Pulmonary edema is associated initially with a dry cough and responds to increased alveolar pressures resulting from continuous positive airway pressure probably due to a reduction in alveolar fluids $[1,18,19]$.

The term pulmonary edema connotes a pathological process; high-altitude pulmonary edema can be life threatening yet a transvascular pulmonary filtrate is a normal physiologic process which is cleared by lymphatic flow. Exercise and hypoxia can accelerate transvascular pulmonary filtrate which can be removed by a normal homeostatic increase in lymph flow that can rise as high as $7-10$ times the baseline level [15]. The episodes of transient dry cough described in this report probably represent the extreme end of the bell shaped curve with elevated pulmonary pressures and hypoxia leading to a prominent transpulmonary filtrate formation with transient fluid build-up due to delayed lymphatic clearance caused by a rapid decrease in the rate and depth of breathing following the sudden cessation of exercise in fit individuals. Clinical pulmonary edema develops when there is a breakdown of the normal homeostatic processes for removal of interstitial fluid perhaps due to a stress failure mechanism [20]; for individuals in this report, the normal homeostatic processes were operative. In summary, non-suppressible dry cough that occurs after intense exercise at altitude and resolves with 20 - 30 minutes of rest probably represents the extreme of normal physiologic processes, does not imply an increased risk of AMS or HAPE, does not require treatment and does not necessitate a restriction in activity or a prohibition against higher ascent.

\section{Conflict of Interest}

None.

\section{Grant Support}

None.

\section{References}

1. Hackett PH, Roach RC. High-altitude illness. N Engl J Med. 2001;345(2):107-114.

2. Taylor A. High-altitude illnesses: Physiology, risk factors, prevention and treatment. RMMJ 2011; 2:1-18.

3. Luks AM, McIntosh SE, Grissom CK, Auerbach PS, Rodway GW, Schoene RB, Zafren K, et al. Wilderness Medical Society consensus guidelines for the prevention and treatment of acute altitude illness. Wilderness Environ Med. 2010;21(2):146-155.

4. Mason NP, Barry PW. Altitude-related cough. Pulm 
Pharmacol Ther. 2007;20(4):388-395.

5. Banner AS, Green J, O'Connor M. Relation of respiratory water loss to coughing after exercise. $\mathrm{N}$ Engl J Med. 1984;311(14):883-886.

6. Zavorsky GS. Evidence of pulmonary oedema triggered by exercise in healthy humans and detected with various imaging techniques. Acta Physiol (Oxf). 2007;189(4):305-317.

7. Hopkins SR. Point: Pulmonary edema does occur in human athletes performing heavy sea-level exercise. J Appl Physiol. 2010;109(4):1270-1272.

8. Roach RC, Maes D, Sandoval D, Robergs RA, Icenogle M, Hinghofer-Szalkay H, Lium D, et al. Exercise exacerbates acute mountain sickness at simulated high altitude. J Appl Physiol. 2000;88(2):581-585.

9. Laffey JG, Kavanagh BP. Hypocapnia. N Engl J Med. 2002;347(1):43-53.

10. Flamm SD, Taki J, Moore R, Lewis SF, Keech F, Maltais F, Ahmad M, et al. Redistribution of regional and organ blood volume and effect on cardiac function in relation to upright exercise intensity in healthy human subjects. Circulation. 1990;81(5):1550-1559.

11. Bartsch P, Mairbaurl H, Maggiorini M, Swenson ER. Physiological aspects of high-altitude pulmonary edema. J Appl Physiol. 2005;98(3):1101-1110.

12. Cremona G, Asnaghi R, Baderna P, Brunetto A, Brutsaert T, Cavallaro C, Clark TM, et al. Pulmonary extravascular fluid accumulation in recreational climbers: a prospective study. Lancet. 2002;359(9303):303-309.

13. Reeves JT, Taylor AE, Rowell LB, Sheperd JT. Pulmonary hemodynamics and fluid exchange in the lungs during exercise. In: Handbook of physiology, section 12: exercise, regulation and intergration of multiple systems. New York:Oxford University Press;1996, p585$613 \mathrm{http} / / /$ onlinelibrary.wiley.com/doi/10.1002/cphy. cp120113/pdf.

14. Newman JH, Butka BJ, Parker RE, Roselli RJ. Effect of progressive exercise on lung fluid balance in sheep. $\mathrm{J}$ Appl Physiol. 1988;64(5):2125-2131.

15. Koizumi T, Roselli RJ, Parker RE, Hermo-Weiler CI, Banerjee M, Newman JH. Clearance of filtered fluid from the lung during exercise: role of hyperpnea. Am J Respir Crit Care Med. 2001;163(3 Pt 1):614-618.

16. McCool FD. Global physiology and pathophysiology of cough: ACCP evidence-based clinical practice guidelines. Chest. 2006;129(1 Suppl):48S-53S.

17. Pare PD, Warriner B, Baile EM, Hogg JC. Redistribution of pulmonary extravascular water with positive endexpiratory pressure in canine pulmonary edema. Am Rev Respir Dis. 1983;127(5):590-593.

18. Agostoni P, Caldara G, Bussotti M, Revera M, Valentini M, Gregorini F, Faini A, et al. Continuous positive airway pressure increases haemoglobin $\mathrm{O} 2$ saturation after acute but not prolonged altitude exposure. Eur Heart J. 2010;31(4):457-463.

19. Koch RO, Hinterhuber L, Faulhaber M, Gatterer H, Graupner S, Muenzel K, Burtscher M. A successful therapy of high-altitude pulmonary edema with a CPAP helmet on Lenin Peak. Clin J Sport Med. 2009;19(1):72-73.

20. West JB, Mathieu-Costello O. High altitude pulmonary edema is caused by stress failure of pulmonary capillaries. Int J Sports Med. 1992;13(Suppl 1):S54-58. 